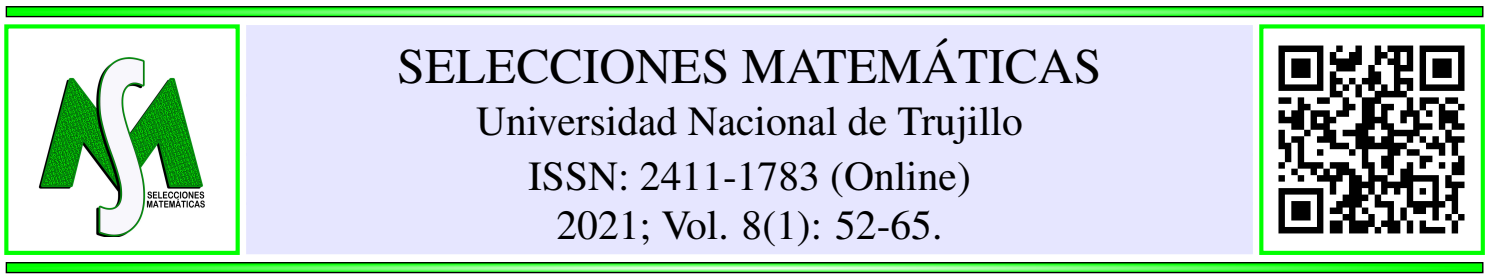

\title{
Lee-Carter method for forecasting mortality for Peruvian Population
}

\author{
J. Cerda-Hernández ${ }^{\circledR}$ and A. Sikov®i
}

Received, Oct. 11, 2020

Accepted, Mar. 14, 2021

How to cite this article:

Cerda-Hernández J. Lee-Carter method for forecasting mortality for Peruvian Population. Selecciones Matemáticas. 2021;8(1):52-65. http://dx.doi.org/10.17268/sel.mat.2021.01.05

\begin{abstract}
In this article, we have modeled mortality rates of Peruvian female and male populations during the period of 1950-2017 using the two-factor Lee-Carter (LC) model. The stochastic mortality model was introduced by Lee and Carter (1992) and has been used by many authors for fitting and forecasting the human mortality rates. The Singular Value Decomposition (SVD) approach is used for estimation of the parameters of the LC model. Utilizing the best fitted auto regressive integrated moving average (ARIMA) model we forecast the values of the time dependent parameter of the LC model for the next thirty years. The forecasted values of life expectancy at different age group with $95 \%$ confidence intervals are also reported for the next thirty years. In this research we use the data, obtained from the Peruvian National Institute of Statistics (INEI).

Keywords . Lee-Carter (LC) model, mortality modeling, forecasting, life expectancy, singular value decomposition (SVD).
\end{abstract}

1. Introduction. Mortality rate is an important variable in the fields of actuarial science, demography, national planning and social security administration. Mortality levels are generally regarded as indicators of a general welfare of a population. Large changes in mortality rates in a relatively short period of time may present a number of challenges to demographers and practitioners of actuarial science. For example, in the Peruavian case the death rate has reduced to a large extent during the last few decades. Specifically, according to the World Health Organization's health statistics 2014, life expectancy at birth has increased by six years between 1990 and 2012 universally (77 years in 2012 as opposed to 71 years in 1990). This arises the need to develop methods for forecasting mortality rates and life expectancy. Prediction of future mortality rates are especially useful for life insurance companies and annuity providers, which use these predicted mortality rates in their pricing calculations. Clearly, systematic underestimation the longevity risk may eventually cause a financial collapse of these companies. For example, if mortality rates increase, the life insurers need to pay the death benefits earlier than expected. This implies that dramatic decline in mortality brings very serious financial exposures for insurers providing life contracts and life annuities.

Lee and Carter [10] introduced the first mortality model with stochastic forecast. The LC model is a two-factor model which includes two age-specific parameters for every age group, and a time-varying effect, such that a tendency of all age-specific central death rates have the same pattern of stochastic evolvement over time. There have been several extensions of the basic Lee-Carter model by including different factors. Both, Maindonald, and Smith [2] considered the multi factor age-period extension of Lee-Carter, Renshaw and Haberman [16] proposed a model with the cohort effect and [4] used the logit transformation in the mortality model, however a good performance of these methods require large number of available observations.

\footnotetext{
*Department of Engineering Economics, National Engineering University, Av. Túpac Amaru, 210, Lima-Perú. (jcerdah@uni.edu.pe).

${ }^{\dagger}$ Department of Engineering Statistics, National Engineering University, Av. Túpac Amaru, 210, Lima-Perú. (asikoveuni.edu.pe).
} 
The main aim of this study is to fit the two-factor LC model for predicting the Peruvian mortality rates and life expectancy in different age groups. This study was motivated by previous research that has shown good performance of the two-factor LC method. For example, these studies include countries such as US [10], Chile [11], China [12], Japan [21], Hungary [1], Singapore [13], Malaysia [14] and India [22], [15]. Also this method is advantageous, when data is available for a short period, which is the main problem in our case. We use life table data from 1950 to 2017. The central mortality rates were measured once during each 5 years period. Based on the LC model, we predict central mortality rates and values of life expectancy at different age groups for the next six periods of five years, starting from the period of 2020-2025.

It must be noticed, however, that due to a very limited number of available observations in the case of Peru, our study heavily relies on good performance of the method, achieved when applied to the data of other countries, mentioned above. In order to evaluate the performance of the model in terms of prediction of the future values, we compare the observed life expectances for each age group to the corresponding predicted values. Standard techniques based on leaving out some data when estimating the model and comparing the predicted values for these data with the observed values are not informative due to a very small number of available observations.

The rest of the paper is organized as follows. In the next section we describe the data obtained from INEI and give a brief discussion of the mortality pattern in Peru. In Section 3 we present the Lee Carter model and describe the estimation and forcasting procedure. In Section 4 we report the results of fitting the Lee Carter model to the Peruvian data. In Section 5 we present the forecasting results. In Section 6 some conclusions are outlined.

2. Data Description. We use five-year age-specific central mortality rates from 1950 to 2017 , available from the Peruvian National Institute of Statistics (INEI). For the peruvian population there are only 14 measurements for each age group: the first measurement refers to the period of 1950-1955, the second measurement refers to the period of 1955-1960, and so on. The last measurement is based on the census, which was conducted in November of 2017 (referred to as a period of 2015-2020). The data is available for 18 age groups: $0,1-4,5-9,10-14, \ldots, 75-79$ and $80+$. Unfortunately, such a layout of the available data is insufficient for deriving some monetary functions involving life contingencies, since this generally requires knowledge of probabilities of death for every single year of age. In the case of mortality at advanced ages the INEI does not have detailed information; the only information available is the central mortality rate at the age group of $80+$. There exist various mortality prediction models for the advanced ages (see for example [6], [18]), however, making analysis of the behavior of mortality rates at advanced ages is not the focus of this research.

The raw data, used for the purpose of implementation of the LC methodology are presented in Tables 5.5 and 5.6

Central Death Rate: Male Population

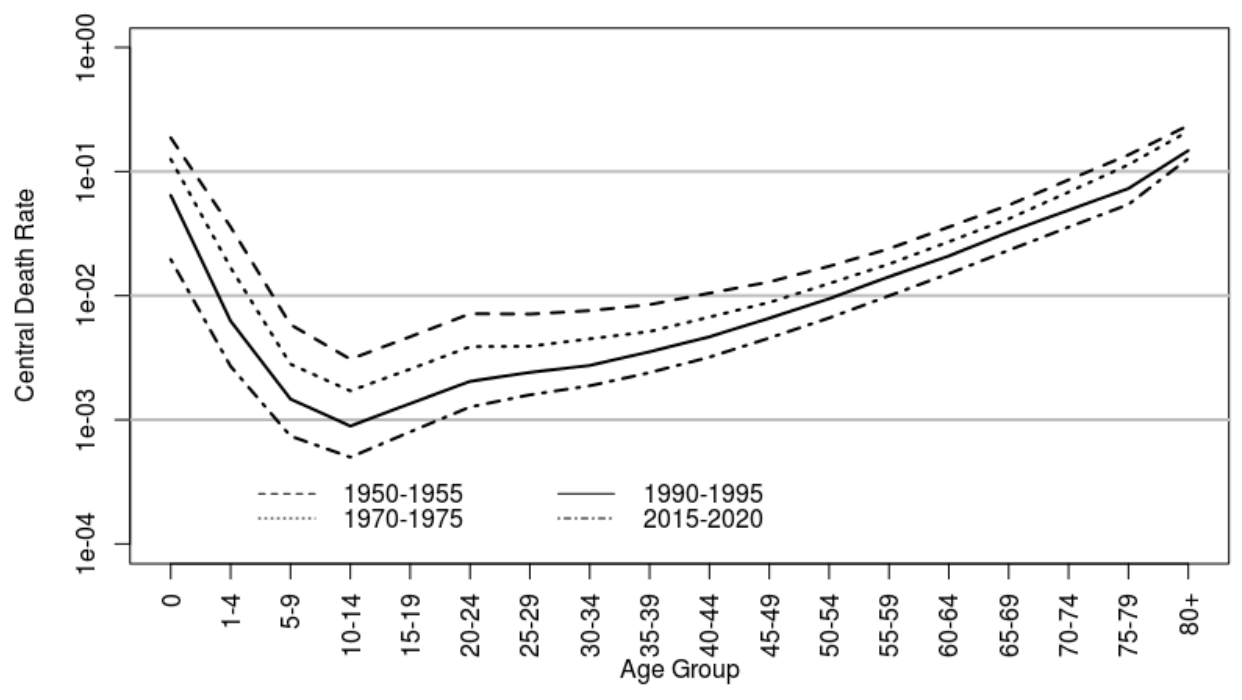

Figure 2.1: The central mortality rates for Peruvian male population. 


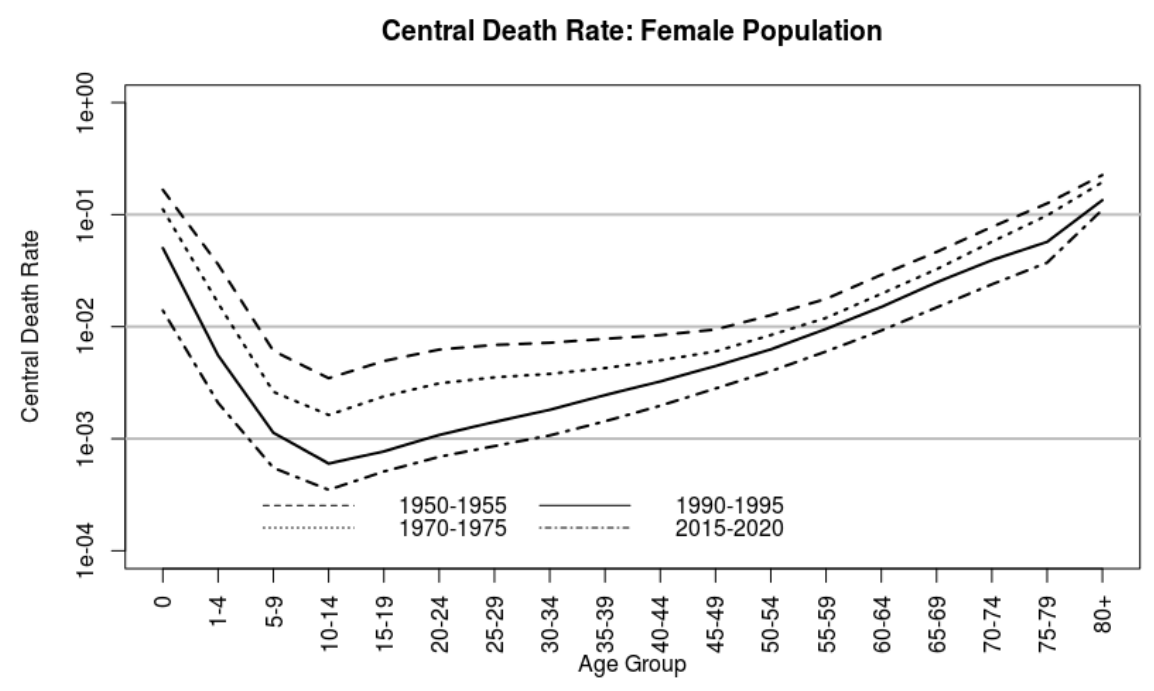

Figure 2.2: The central mortality rates for Peruvian female population.

Figures 2.1 and 2.2 present the age group specific central mortality rates for Peruvian female and male populations for 4 different periods: 1950-1955, 1970-1975, 1990-1995 and 2015-2020. These figures shows very clearly a notable reduction in mortality rates in Peru over time for both male and female populations. One can also observe that a more conciderable decline in mortality rates occurs in the younger ages groups. For the female population we observe a more rapid decline in mortality rates for the age groups from 10 to 40 years, during 1970 to 1995 as compared to the periods between 1950 to 1975 and between 1995-2015, while for the male population for the mentioned periods the decline is uniform. We can also conclude that during the periods between 1950 and 1975 and between 1975 and 1995, a more conciderable reduction occured for the age groups between 5 and 40, compared to the older age groups. During the last period between 1995 and 2015 the decline is generally more or less uniform for all the age groups.

Based on the data on mortality tables we compute life expectancies for female and male populations for all available 5 years periods, for several age groups. The results are presented in Figures 2.3 and 2.4. The figures illustrate an increase in life expectancy for all age groups for both male and female populations: from drastic for the infants (72.5 and 77.8 years in 2017 as opposed to 42.9 and 45.0 in 1950-1955) to quite modest for the 75-79 age group (10.4 and 12.1 in 2017 as opposed to 5.8 and 6.1 in 1950-1955). One can also observe that in the period between 1950 and 1960, life expectancy of Peruvian female and male at birth is quite close to life expectancy in the group of 20-24. This can be explained by high rate of infant mortality during this decade.

3. Lee-Carter Model. For our study there are only 14 measurements available for each age group. Let $m_{x, t}$ denote the central mortality rate for the age group $x$, during the five-years period $t$, where $x \in$ $\{0,1-4,5-9,10-14,15-19,20-24,25-29,30-34,35-39,40-44,45-49,50-54,55-$ $59,60-64,65-69,70-74,75-79,80+\}$ and $t \in\{1950-1955,1955-1960,1960-1965,1965-$ $1970,1970-1975,1975-1980,1980-1985,1985-1990,1990-1995,1995-2000,2000-2005,2005-$ $2010,2010-2015,2015-2020\}$.

The two-factor LC model uses the natural logarithm of the central mortality rates to measure the age and time effect, and is defined as

$$
r_{x, t}=\ln \left(m_{x, t}\right)=\alpha_{x}+\beta_{x} k_{t}+\varepsilon_{x, t},
$$

where $\alpha_{x}$ denotes the coefficient which describes average age specific pattern by age of mortality, $k_{t}$ denotes the time-varying index for the general mortality, $\beta_{x}$ denotes the coefficient which measures sensitivity of $\ln \left(m_{x, t}\right)$ at age group $x$ to changing the index $k_{t}$ (note that in a continuous contex we have $d \ln \left(m_{x, t}\right) / d t=$ $\left.\beta_{x} d k_{t} / d t\right)$ and $\varepsilon_{x, t}$ is the error term that represent age specific historical influences not captured by the model. We assume that it follows a normal distribution with mean zero and variance $\sigma_{\varepsilon}^{2}$, and that the errors are independent of age group and time, that is, $\varepsilon_{x, t} \sim \operatorname{IIDN}\left(0, \sigma_{\varepsilon}^{2}\right)$. The term $\beta_{x} k_{t}$ in the LC model capture the joint tendency of age-specific mortality rates to evolve over time. 


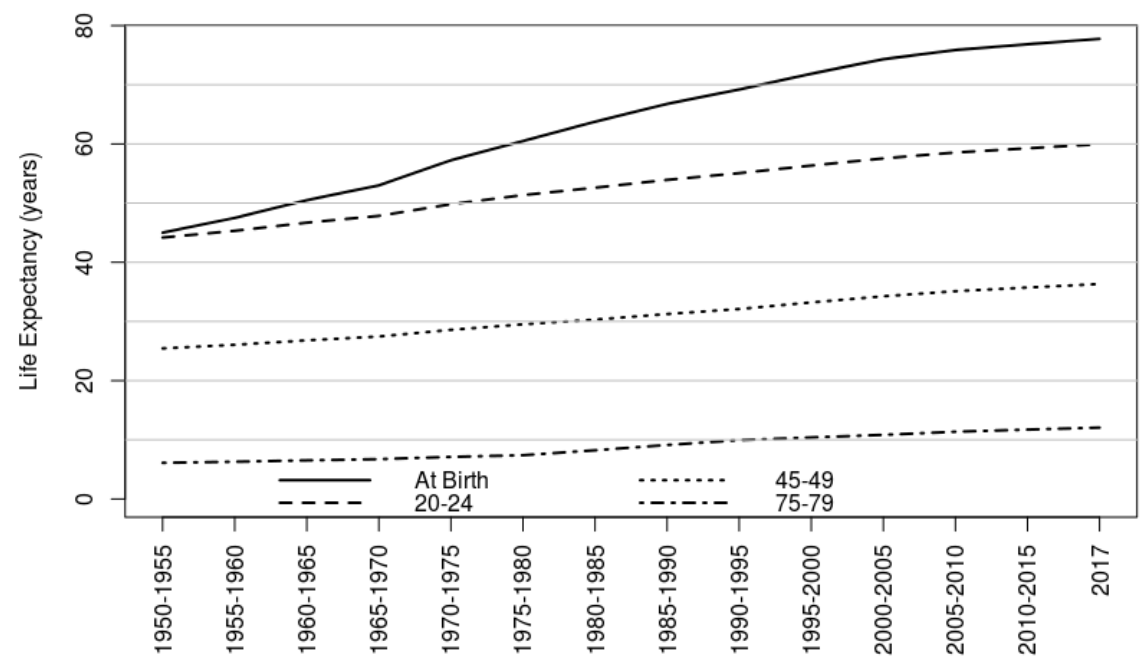

Figure 2.3: Life expectancy (in years) of Peruvian female population during 1950-2017 at selected age groups

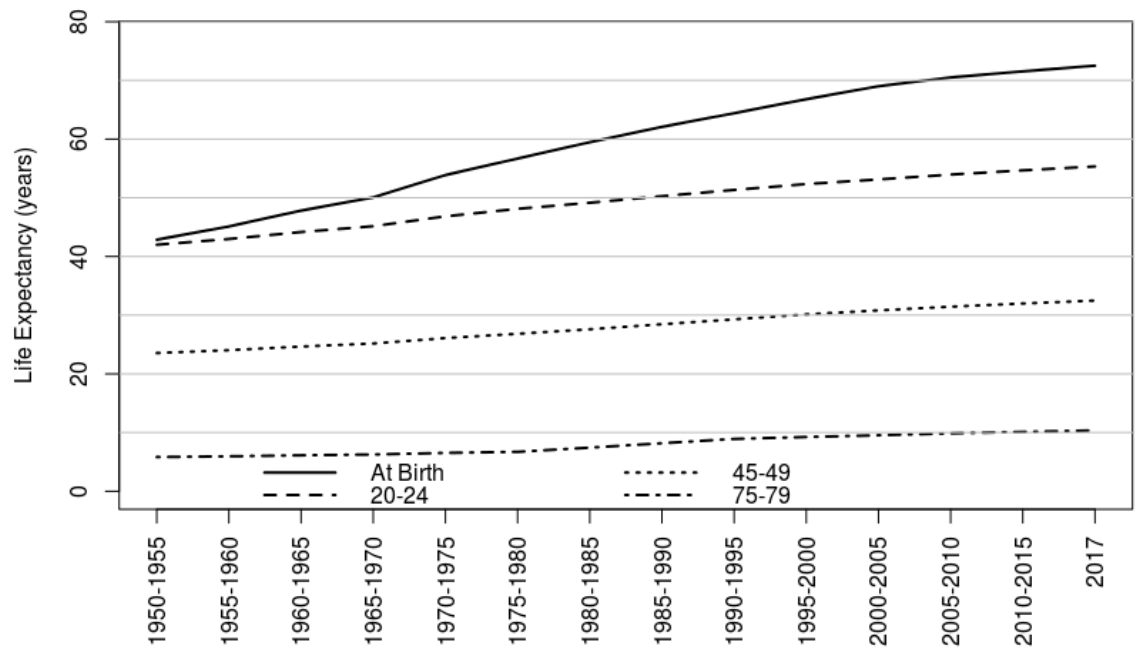

Figure 2.4: Life expectancy (in years) of Peruvian male population during 1950-2017 at selected age groups

The model can not be adjusted by regression methods since no explanation variables are included into the model. Moreover, the model is not identifiable (see Lee and Carter, 1992). In order to solve this problem, the authors use the following constraints: $\sum_{t} k_{t}=0$ and $\sum_{x} \beta_{x}=1$.

The first constraint can be rewritten as

$$
\begin{aligned}
\frac{1}{T} \sum_{t=1}^{T} \ln \left(m_{x, t}\right) & =\alpha_{x}+\beta_{x} \frac{1}{T} \sum_{t=1}^{T} k_{t}+\frac{1}{T} \sum_{t=1}^{T} \varepsilon_{x, t} \\
& =\alpha_{x}+\frac{1}{T} \sum_{t=1}^{T} \varepsilon_{x, t} .
\end{aligned}
$$

Note that the expression above implies that

$$
\alpha_{x}=\mathbb{E}\left[\frac{1}{T} \sum_{t=1}^{T} \ln \left(m_{x, t}\right)\right]
$$


for all $x$, and if $T$ gets large, by the Strong laws of large numbers, we have that $\frac{1}{T} \sum_{t=1}^{T} \varepsilon_{x, t}$ converge almost surely (a.s.) to 0 . Thus, for all $x$, a natural estimator for the parameter $\alpha_{x}$ is equal to the average of $\ln \left(m_{x, t}\right)$ over time, that is,

$$
\hat{\alpha}_{x}=\frac{1}{T} \sum_{t=1}^{T} \ln \left(m_{x, t}\right),
$$

where $T$ is the number of available time periods (recall that in our case, $T=14$ ). We therefore rewrite the model in terms of the mean centered log-mortality rate, $\widetilde{r}_{x, t}=r_{x, t}-\alpha_{x}$. Since practical uses of the LC model implicitly assume that the errors $\varepsilon_{x, t}$ are normally distributed, the Equation (3.1) can be expressed as a multiplicative fixed effects model for the centered age profile:

$$
\begin{aligned}
& \tilde{r}_{x, t} \sim N\left(\beta_{x} k_{t}, \sigma^{2}\right), \\
& \tilde{r}_{x, t}=\beta_{x} k_{t}+\varepsilon_{x, t},
\end{aligned}
$$

where the parameter $\mathbb{E}\left(\widetilde{r}_{x, t}\right)=\beta_{x} k_{t}$ is interpreted as the average pattern of mortality at age group $x$ and time period $t$. Using the second constraint of the model, we obtain an estimate of $k_{t}$ given by

$$
\hat{k}_{t}=\sum_{x} \ln \left(m_{x, t}\right)-\hat{\alpha}_{x}
$$

Differentiating both sides of (3.1) we obtain an estimate for $\beta_{x}, \hat{\beta}_{x}=\left(\partial \ln \left(m_{x, t}\right) / \partial t\right) /\left(\partial k_{t} / \partial t\right)$.

Following Lee and Carter (1992), the estimators for $\beta_{x}$ and $k_{t}$ can be obtained by using the Singular Value Decomposition of the matrix $\mathbf{M}=\left\{M_{x, t}=\ln \left(m_{x, t}\right)-\hat{\alpha}_{x}\right\}_{x, t}$ (see [9], [17] for more details). Singular Value Decomposition establish that $\mathbf{M}$ can be written as follows

$$
\operatorname{svd}(\mathbf{M})_{x, t}=\sum_{i=1}^{q} \lambda_{i} U_{x, i} V_{i, t},
$$

where $q=\operatorname{rank}(\mathbf{M}),\left\{\lambda_{1} \geq \lambda_{2} \geq \cdots \geq \lambda_{q}\right\}$ are the ordered singular values of $\mathbf{M}$, and columns of $U$ and the columns of $V$ are called the left-singular vectors and right-singular vectors of $\mathbf{M}$, respectively. In our case the dimension of $\mathbf{M}$ is $18 \times 14$. Utilizing the Theorem of low rank approximation, the rank- $h$ least square approximation of (3.3) is obtained as

$$
M_{x, t}^{(h)}=\sum_{i=1}^{h} \lambda_{i} U_{x, i} V_{i, t}=\sum_{i=1}^{h} \beta_{x}^{(i)} k_{t}^{(i)}, \quad h \leq q,
$$

where $\beta_{x}^{(i)} k_{t}^{(i)}=\lambda_{i} U_{x, i} V_{i, t}$ (see [9], [17] and [8] for more details and properties of SVD). Then, the rank- $h$ residuals associated with (3.4) are

$$
\varepsilon_{x, t}=\sum_{i=h+1}^{r} \lambda_{i} U_{x, i} V_{i, t}=U\left[\begin{array}{ccccccc}
0 & \cdots & 0 & \cdots & 0 & \cdots & 0 \\
\vdots & \ddots & \vdots & \ddots & \vdots & \ddots & \vdots \\
0 & \cdots & \lambda_{h+1} & \cdots & 0 & \cdots & 0 \\
\vdots & \ddots & \vdots & \ddots & \vdots & \ddots & \vdots \\
0 & \cdots & 0 & \cdots & \lambda_{r} & \cdots & 0
\end{array}\right] V
$$

and the corresponding rank- $h$ approximation least square errors is

$$
\varepsilon_{h}^{2}=\sum_{i=h+1}^{r} \lambda_{i}^{2}
$$

which implies that the errors have similar variance. However, this assumption is violated for mortality data. For example, in [20] the authors show that the variance of the log-central death rate is approximately $\operatorname{Var}\left(\ln \left(m_{x, t}\right)\right) \approx 1 / d_{x, t}$, where $d_{x, t}$ denote the number of deaths at the age group $x$ at time $t$.

The proportion of variance explained by the $i^{t h}$ term $\lambda_{i} U_{x, i} V_{i, t}$ of the decomposition (3.3) is given by the expression $\lambda_{i}^{2} / \sum_{j=1}^{r} \lambda_{j}^{2}$, and the total variance explained by the rank- $h$ approximation is given by

$$
\sigma_{h}^{2}=\sum_{i=1}^{h} \lambda_{i}^{2} / \sum_{j=1}^{r} \lambda_{j}^{2}
$$


It is clear that $0 \leq \sigma_{h}^{2} \leq 1$ and the closer this value is to 1 , the better is the approximation. For example, for the US data, Lee and Carter [10] restrained the SVD approximation to the first order $M_{x, t}^{(1)} \approx \lambda_{1} U_{x, 1} V_{1, t}=$ $\beta_{x}^{(1)} k_{t}^{(1)}$ and obtained an explained variance $\sigma_{1}^{2}=92.7 \%$ for the total population. In our case, application of the first order approximation, yields even better percentages of explained variances: $98.73 \%$ and $98.77 \%$ corresponding to the male and female populations, respectively.

Finally, predicting mortality with the two factor LC model is reduced to forecasting the time-varying index $k_{t}$ for the general mortality utilizing time series approaches (see for example [5] and [3]).

4. Fitting of the LC Model to Peruvian Mortality Data. This section presents the results of estimation of parameters in LC model, described in the previous section, for female and male populations of Peru, based on mortality tables available from the period of 1950-55 to the period of 2015-20. The estimated values of age dependent parameters $\alpha_{x}$ and $\beta_{x}$ are reported in Table 4.1 and the estimated values of time dependent parameter $k_{t}$ are reported in Table 4.2

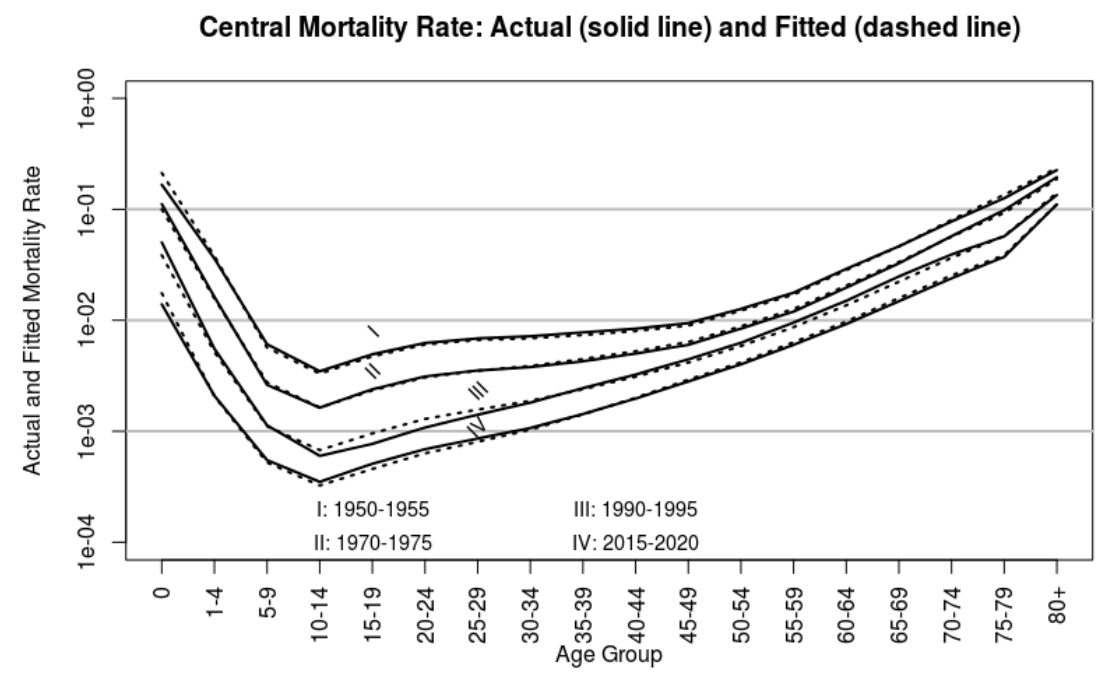

Figure 4.1: Actual and Fitted age group specific central mortality rates for Peruvian female population.

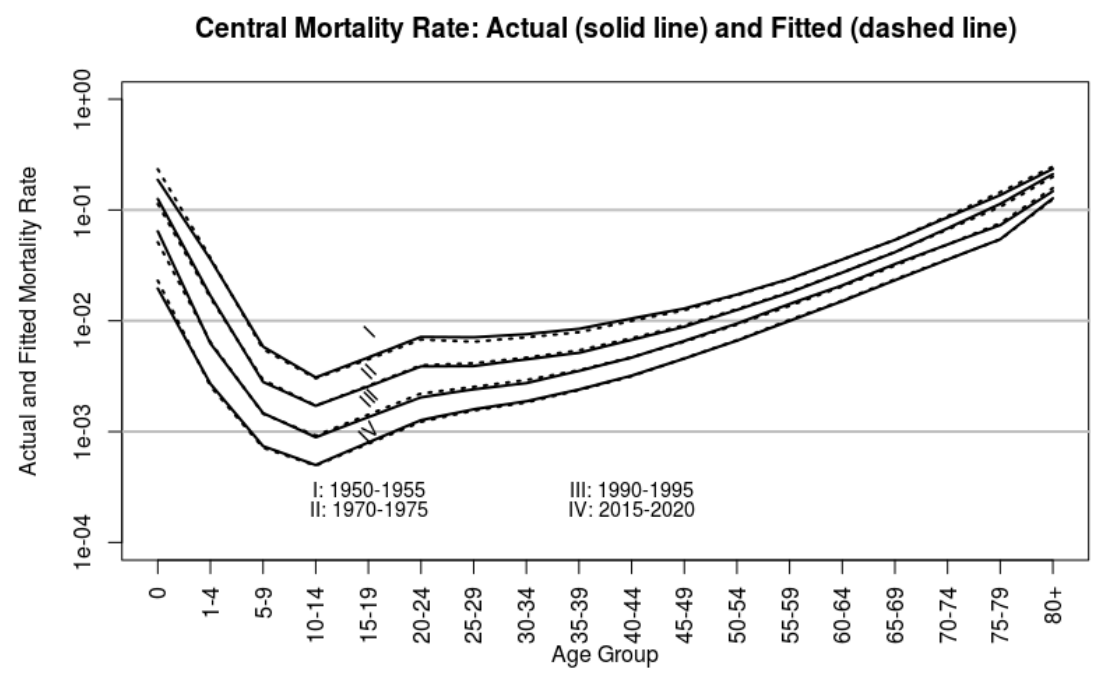

Figure 4.2: Actual and Fitted age group specific central mortality rates for Peruvian male population.

Applying SVD to the matrix $M_{x, t}$, we obtained an explained variance of $98.73 \%$ and $98.77 \%$ by fitted LC model for Peruvian female and male mortality data respectively. In Figures 4.1 and 4.2, we have plotted 
the observed and fitted age group specific central mortality rates for four periods: 1950-1955, 1970-1975, 1990-1995 and 2015-2020. The obtained results indicate that the fitted mortality rates, obtained by fitting the LC model are generally very close to the observed (actual) mortality rates for both male and female populations although for the period of 1990-1995, the estimated mortality rates for females of the age groups 15-19 and 20-24 are somewhat higher than the actual mortality rates. Also there are some small differences for the newborns.

In Tables 4.3 and 4.4, we present the actual values of LE and their estimated values, based on fitted LC for four selected decades.

\begin{tabular}{|c|c|c|c|c|}
\hline \multirow{2}{*}{$\begin{array}{c}\text { Age } \\
\text { Group }\end{array}$} & \multicolumn{2}{|c|}{ Female } & \multicolumn{2}{c|}{ Male } \\
\cline { 2 - 5 } & $\hat{\alpha}_{x}$ & $\hat{\beta}_{x}$ & $\hat{\alpha}_{x}$ & $\hat{\beta}_{x}$ \\
\hline 0 & -2.8535 & 0.0825 & -2.6463 & 0.0946 \\
\hline $1-4$ & -4.7967 & 0.0963 & -4.6714 & 0.1092 \\
\hline $5-9$ & -6.4206 & 0.0789 & -6.2496 & 0.0838 \\
\hline $10-14$ & -6.9271 & 0.0768 & -6.7344 & 0.0741 \\
\hline $15-19$ & -6.5770 & 0.0772 & -6.3082 & 0.0717 \\
\hline $20-24$ & -6.2901 & 0.0747 & -5.8756 & 0.0699 \\
\hline $25-29$ & -6.1192 & 0.0699 & -5.7764 & 0.0585 \\
\hline $30-34$ & -5.9718 & 0.0627 & -5.6453 & 0.0554 \\
\hline $35-39$ & -5.7718 & 0.0545 & -5.4624 & 0.0492 \\
\hline $40-44$ & -5.5531 & 0.0459 & -5.1991 & 0.0470 \\
\hline $45-49$ & -5.3026 & 0.0371 & -4.9001 & 0.0408 \\
\hline $50-54$ & -4.9664 & 0.0355 & -4.5534 & 0.0382 \\
\hline $55-59$ & -4.5889 & 0.0327 & -4.1751 & 0.0345 \\
\hline $60-64$ & -4.1227 & 0.0354 & -3.7713 & 0.0348 \\
\hline $65-69$ & -3.6327 & 0.0355 & -3.3433 & 0.0336 \\
\hline $70-74$ & -3.1249 & 0.0382 & -2.8910 & 0.0367 \\
\hline $75-79$ & -2.6545 & 0.0414 & -2.4385 & 0.0403 \\
\hline $80+$ & -1.8416 & 0.0249 & -1.7508 & 0.0278 \\
\hline
\end{tabular}

Table 4.1: Estimates of $\alpha_{x}$ and $\beta_{x}$ for Peruvian population based on quinquennial-wise mortality tables (1950-55 to 2015-20)

\begin{tabular}{|c|c|c|c|c|c|c|c|}
\hline$t$ & $1950-55$ & $1955-60$ & $1960-65$ & $1965-70$ & $1970-75$ & $1975-80$ & $1980-85$ \\
\hline$\hat{k}_{t}($ female $)$ & 15.826 & 14.176 & 12.082 & 10.216 & 6.697 & 3.648 & 0.777 \\
$\hat{k}_{t}$ (male) & 12.598 & 11.193 & 9.430 & 7.864 & 4.980 & 2.637 & 0.606 \\
\hline \hline$t$ & $1985-90$ & $1990-95$ & $1995-00$ & $2000-05$ & $2005-10$ & $2010-15$ & $2015-20$ \\
\hline$\hat{k}_{t}$ (female) & -2.195 & -4.841 & -7.277 & -9.920 & -11.753 & -13.043 & -14.391 \\
$\hat{k}_{t}$ (male) & -1.448 & -3.419 & -5.463 & -7.473 & -9.148 & -10.504 & -11.853 \\
\hline
\end{tabular}

Table 4.2: $k_{t}$ for Peruvian population based on quinquennial wise mortality tables (1950-55 to 2015-20) 


\begin{tabular}{|l|l|l|l|l|l|l|l|l|}
\hline \multirow{2}{*}{$\begin{array}{c}\text { Age } \\
\text { Group }\end{array}$} & \multicolumn{2}{|c|}{$1950-1955$} & \multicolumn{2}{c|}{$1970-1975$} & \multicolumn{2}{c|}{$1990-1995$} & \multicolumn{2}{c|}{$2015-2020$} \\
\cline { 2 - 9 } & Observed & Estimated & Observed & Estimated & Observed & Estimated & Observed & Estimated \\
\hline 0 & 42.57 & 40.79 & 53.73 & 54.42 & 64.33 & 64.94 & 72.49 & 72.32 \\
\hline $1-4$ & 50.24 & 50.39 & 59.88 & 59.90 & 67.57 & 67.33 & 72.91 & 73.00 \\
\hline $5-9$ & 53.67 & 54.13 & 59.91 & 59.76 & 65.23 & 65.04 & 69.68 & 69.73 \\
\hline $10-19$ & 50.19 & 50.58 & 55.72 & 55.61 & 60.70 & 60.49 & 64.93 & 64.97 \\
\hline $15-19$ & 45.93 & 46.31 & 51.18 & 51.07 & 55.95 & 55.76 & 60.09 & 60.12 \\
\hline $20-24$ & 41.96 & 42.31 & 46.81 & 46.70 & 51.32 & 51.14 & 55.32 & 55.35 \\
\hline $25-29$ & 38.40 & 38.68 & 42.68 & 42.59 & 46.82 & 46.68 & 50.65 & 50.67 \\
\hline $30-34$ & 34.70 & 34.87 & 38.47 & 38.43 & 42.35 & 42.25 & 46.04 & 46.05 \\
\hline $35-39$ & 30.94 & 31.04 & 34.29 & 34.28 & 37.90 & 37.83 & 41.45 & 41.45 \\
\hline $40-44$ & 27.17 & 27.19 & 30.11 & 30.15 & 33.54 & 33.47 & 36.92 & 36.91 \\
\hline $45-49$ & 23.50 & 23.46 & 26.06 & 26.13 & 29.27 & 29.21 & 32.47 & 32.46 \\
\hline $50-54$ & 19.90 & 19.80 & 22.12 & 22.24 & 25.16 & 25.09 & 28.17 & 28.16 \\
\hline $55-59$ & 16.47 & 16.35 & 18.39 & 18.54 & 21.26 & 21.16 & 24.03 & 24.03 \\
\hline $60-64$ & 13.25 & 13.10 & 14.89 & 15.07 & 17.64 & 17.48 & 20.13 & 20.16 \\
\hline $65-69$ & 10.37 & 10.18 & 11.71 & 11.93 & 14.31 & 14.09 & 16.51 & 16.56 \\
\hline $70-74$ & 7.83 & 7.60 & 8.86 & 9.14 & 11.41 & 11.08 & 13.24 & 13.34 \\
\hline $75-79$ & 5.78 & 5.52 & 6.50 & 6.82 & 8.90 & 8.49 & 10.35 & 10.49 \\
\hline $80+$ & 4.26 & 4.06 & 4.74 & 5.01 & 6.78 & 6.33 & 7.84 & 8.01 \\
\hline
\end{tabular}

Table 4.3: Observed and estimated life expectancy for the periods of 1950-1955, 1970-1975, 1990-1995 and 2015-2020, Males

\begin{tabular}{|l|l|l|l|l|l|l|l|l|}
\hline \multirow{2}{*}{$\begin{array}{c}\text { Age } \\
\text { Group }\end{array}$} & \multicolumn{2}{|c|}{$1950-1955$} & \multicolumn{2}{c|}{$1970-1975$} & \multicolumn{2}{c|}{$1990-1995$} & \multicolumn{2}{c|}{$2015-2020$} \\
\cline { 2 - 9 } & Observed & Estimated & Observed & Estimated & Observed & Estimated & Observed & Estimated \\
\hline 0 & 44.76 & 42.75 & 57.13 & 57.75 & 69.15 & 70.25 & 77.75 & 77.22 \\
\hline $1-4$ & 51.79 & 51.77 & 62.83 & 62.78 & 71.70 & 72.00 & 77.84 & 77.58 \\
\hline $5-9$ & 55.50 & 55.93 & 62.91 & 62.73 & 69.26 & 69.47 & 74.48 & 74.21 \\
\hline $10-14$ & 52.13 & 52.47 & 58.71 & 58.57 & 64.64 & 64.84 & 69.67 & 69.40 \\
\hline $15-19$ & 48.00 & 48.30 & 54.17 & 54.03 & 59.82 & 60.05 & 64.79 & 64.51 \\
\hline $20-24$ & 44.14 & 44.40 & 49.79 & 49.64 & 55.05 & 55.33 & 59.95 & 59.65 \\
\hline $25-29$ & 40.46 & 40.68 & 45.53 & 45.36 & 50.33 & 50.67 & 55.15 & 54.83 \\
\hline $30-34$ & 36.79 & 36.97 & 41.30 & 41.12 & 45.67 & 46.05 & 50.38 & 50.04 \\
\hline $35-39$ & 33.05 & 33.18 & 37.04 & 36.88 & 41.06 & 41.46 & 45.63 & 45.29 \\
\hline $40-44$ & 29.26 & 29.33 & 32.79 & 32.66 & 36.54 & 36.93 & 40.94 & 40.59 \\
\hline $45-49$ & 25.41 & 25.43 & 28.56 & 28.46 & 32.09 & 32.47 & 36.32 & 35.98 \\
\hline $50-54$ & 21.52 & 21.48 & 24.35 & 24.31 & 27.76 & 28.10 & 31.80 & 31.47 \\
\hline $55-59$ & 17.76 & 17.68 & 20.29 & 20.29 & 23.56 & 23.86 & 27.39 & 27.08 \\
\hline
\end{tabular}




\begin{tabular}{|l|l|l|l|l|l|l|l|l|}
\hline $60-64$ & 14.18 & 14.03 & 16.39 & 16.45 & 19.59 & 19.81 & 23.15 & 22.87 \\
\hline $65-69$ & 11.01 & 10.80 & 12.83 & 12.97 & 15.93 & 16.03 & 19.13 & 18.89 \\
\hline $70-74$ & 8.26 & 7.99 & 9.67 & 9.89 & 12.71 & 12.63 & 15.42 & 15.25 \\
\hline $75-79$ & 6.07 & 5.79 & 7.09 & 7.36 & 9.93 & 9.68 & 12.06 & 11.98 \\
\hline $80+$ & 4.43 & 4.25 & 5.13 & 5.34 & 7.43 & 7.11 & 9.03 & 9.02 \\
\hline
\end{tabular}

Table 4.4: Observed and estimated life expectancy for the periods of 1950-1955, 1970-1975, 1990-1995 and 2015-2020, Females

The results presented in Tables 4.3 and 4.4 show a very good fit of the LC model to the data for both female and male populations, for all age groups.

5. Forecasting. Forecasting is generally the main aim behind the modeling of mortality rates. The notable advantage of the LC model is its simplicity for predicting the future values of central mortality rates and life expectancy, since the values of the coefficients $a_{x}$ and $\beta_{x}$ are supposed to be constant over time. It follows then that in order to predict the future values of the mortality rate (and the life expectancy) one has to predict the corresponding value of the mortality index $k_{t}$. Following other authors (Lee and Carter (1992), Chavhan and Shinde (2016) among many others), we fit an arima model to the serie $k_{t}$. Having fitted an appropriate model to the series of the observed values of $k_{t}$, one can predict its future values, and consequently, compute predictions of the age specific central mortality rates and life expectancy, using the obtained values of $a_{x}$ and $b_{x}$ (see table 4.1).

Following Lee and Carter (1992), we considered a variety of ARIMA models to be fitted to mortality index for male and female populations. In both cases the best fitted model was the ARIMA(0,2,0). As a selection criteria we used the Akaike's information criterion (AIC). Next, we checked normality with Kolmogorov-Smirnov test, and absence of autocorrelations of the model residuals. The following tables present the results of estimation and forecasting procedures. In Table 5.1, we report the predicted values of mortality index, along with their corresponding standard errors for the next six periods (from 2020-2025 to 2045-2050). The results presented in the table show a steady reduction in predicted mortality rates over time for all age groups for both males and females. Also, one can observe that a more rapid reduction occurs in the younger age groups. For example, for the newborns, a reduction is about $43 \%$ (from 1573 in 2020-2025 to 902 in 2045-2050) for females and about 47\% for males (from 2034 in 2020-2025 to 1074 in 2045-2050); for the age group 45-49 a reduction is $22 \%$ and $24 \%$ for females and males correspondingly, and for the age group of $80+$ a reduction is quite modest: $15 \%$ and $17 \%$.

\begin{tabular}{|c|c|c|c|c|c|c|}
\hline$t$ & $2020-2025$ & $2025-2030$ & $2030-2035$ & $2035-2040$ & $2040-2045$ & $2045-2050$ \\
\hline$\hat{k}_{t}$ (female) & $-15.740(0.605)$ & $-17.088(1.353)$ & $-18.436(2.263)$ & $-19.784(3.313)$ & $-21.132(4.486)$ & $-22.480(5.770)$ \\
$\hat{k}_{t}$ (male) & $-13.203(0.459)$ & $-14.552(1.026)$ & $-15.902(1.717)$ & $-17.251(2.513)$ & $-18.600(3.402)$ & $-19.950(4.376)$ \\
\hline
\end{tabular}

Table 5.1: Predicted values of mortality index $k_{t}$ with standard errors (in the parenteses), based on ARIMA $(0,2,0)$ model for the next 6 quinnquenials (2020-2025 to 2045-2050)

Table 5.2 presents the forecasted age specific central death rates in terms of deaths per 100,000 for the next six periods.

\begin{tabular}{|c|c|c|c|c|c|c|c|c|c|c|c|c|}
\hline \multirow{2}{*}{$\begin{array}{c}\text { Age } \\
\text { Group }\end{array}$} & \multicolumn{6}{|c|}{ Female } & \multicolumn{6}{|c|}{ Male } \\
\hline & I & II & III & IV & $\mathrm{V}$ & VI & I & II & III & IV & V & VI \\
\hline 0 & 1573 & 1407 & 1259 & 1127 & 1008 & 902 & 2034 & 1790 & 1575 & 1387 & 1220 & 1074 \\
\hline $1-4$ & 181 & 159 & 140 & 123 & 108 & 95 & 221 & 191 & 165 & 142 & 123 & 106 \\
\hline $5-9$ & 47 & 42 & 38 & 34 & 31 & 28 & 64 & 57 & 51 & 46 & 41 & 36 \\
\hline
\end{tabular}




\begin{tabular}{|l|l|l|l|l|l|l|l|l|l|l|l|l|}
\hline $10-14$ & 29 & 26 & 24 & 21 & 19 & 17 & 45 & 40 & 37 & 33 & 30 & 27 \\
\hline $15-19$ & 41 & 37 & 34 & 30 & 27 & 25 & 71 & 64 & 58 & 53 & 48 & 44 \\
\hline $20-24$ & 57 & 52 & 47 & 42 & 38 & 35 & 112 & 102 & 92 & 84 & 77 & 70 \\
\hline $25-29$ & 73 & 67 & 61 & 55 & 50 & 46 & 143 & 132 & 122 & 113 & 104 & 96 \\
\hline $30-34$ & 95 & 87 & 80 & 74 & 68 & 62 & 170 & 158 & 147 & 136 & 126 & 117 \\
\hline $35-39$ & 132 & 123 & 114 & 106 & 99 & 92 & 222 & 208 & 194 & 182 & 170 & 159 \\
\hline $40-44$ & 188 & 177 & 166 & 156 & 147 & 138 & 297 & 279 & 261 & 245 & 230 & 216 \\
\hline $45-49$ & 278 & 264 & 251 & 239 & 227 & 216 & 434 & 411 & 389 & 368 & 348 & 330 \\
\hline $50-54$ & 399 & 380 & 362 & 346 & 329 & 314 & 636 & 604 & 574 & 545 & 518 & 492 \\
\hline $55-59$ & 607 & 581 & 556 & 532 & 509 & 487 & 975 & 930 & 888 & 848 & 809 & 772 \\
\hline $60-64$ & 928 & 885 & 844 & 804 & 767 & 731 & 1453 & 1387 & 1323 & 1262 & 1204 & 1149 \\
\hline $65-69$ & 1513 & 1442 & 1375 & 1310 & 1249 & 1191 & 2266 & 2166 & 2070 & 1978 & 1890 & 1806 \\
\hline $70-74$ & 2410 & 2289 & 2175 & 2066 & 1962 & 1864 & 3422 & 3257 & 3100 & 2950 & 2808 & 2672 \\
\hline $75-79$ & 3664 & 3465 & 3276 & 3098 & 2930 & 2771 & 5131 & 4859 & 4602 & 4359 & 4129 & 3910 \\
\hline $80+$ & 10717 & 10363 & 10021 & 9690 & 9371 & 9061 & 12028 & 11585 & 11159 & 10748 & 10352 & 9971 \\
\hline
\end{tabular}

Table 5.2: Forecasted values of age specific mortality rates in terms per 100,000 for 2020-2025 (I), 20252030 (II), 2030-2035 (III), 2035-2040 (IV), 2040-2045 (V) and 2045-2050 (VI)

Finally, Tables 5.3 and 5.4 present the forecasted values of life expectancy and the corresponding $95 \%$ confidence intervals. From these tables one can observe that life expectancy at birth will increase from 77.75 to 81.98 for females and from 72.49 to 77.83 for males (between the periods of 2015-2020 and 20452050). However, as one can see, the width of confidence intervals significantly increases for more distant periods. For example, for the period of 2045-2050 a confidence bands are around 4 years width for females and about 10 years width for males. For the period of 2020-2025 the band width is quite narrow: around 1 year for females and around 5 years for males. For the younger age groups the confidence bands are generally wider.

\begin{tabular}{|c|c|c|c|c|c|c|c|c|c|c|c|c|}
\hline \multirow{2}{*}{$\begin{array}{l}\text { Age } \\
\text { Group }\end{array}$} & \multicolumn{6}{|c|}{ Female } & \multicolumn{6}{|c|}{ Male } \\
\hline & I & II & III & IV & $\mathrm{V}$ & VI & I & II & III & IV & $\mathrm{V}$ & VI \\
\hline 0 & 78.07 & 78.88 & 79.68 & 80.46 & 81.23 & 81.98 & 73.31 & 74.27 & 75.20 & 76.10 & 76.97 & 77.83 \\
\hline $1-4$ & 78.30 & 79.00 & 79.69 & 80.37 & 81.04 & 81.72 & 73.81 & 74.60 & 75.39 & 76.15 & 76.91 & 77.67 \\
\hline $5-9$ & 74.85 & 75.49 & 76.12 & 76.75 & 77.39 & 78.02 & 70.45 & 71.16 & 71.87 & 72.58 & 73.28 & 73.99 \\
\hline $10-14$ & 70.02 & 70.64 & 71.26 & 71.88 & 72.50 & 73.12 & 65.67 & 66.36 & 67.05 & 67.74 & 68.43 & 69.12 \\
\hline $15-19$ & 65.12 & 65.73 & 66.34 & 66.96 & 67.57 & 68.18 & 60.81 & 61.49 & 62.17 & 62.85 & 63.53 & 64.21 \\
\hline $20-24$ & 60.25 & 60.85 & 61.45 & 62.05 & 62.66 & 63.26 & 56.01 & 56.68 & 57.34 & 58.01 & 58.67 & 59.34 \\
\hline $25-29$ & 55.42 & 56.00 & 56.59 & 57.18 & 57.77 & 58.37 & 51.31 & 51.95 & 52.59 & 53.24 & 53.89 & 54.54 \\
\hline $30-34$ & 50.61 & 51.18 & 51.75 & 52.33 & 52.91 & 53.50 & 46.66 & 47.28 & 47.90 & 48.53 & 49.16 & 49.79 \\
\hline $35-39$ & 45.84 & 46.39 & 46.95 & 47.51 & 48.08 & 48.66 & 42.04 & 42.64 & 43.24 & 43.84 & 44.45 & 45.07 \\
\hline $40-44$ & 41.13 & 41.66 & 42.21 & 42.75 & 43.31 & 43.87 & 37.48 & 38.05 & 38.63 & 39.22 & 39.81 & 40.41 \\
\hline $45-49$ & 36.49 & 37.01 & 37.54 & 38.07 & 38.61 & 39.16 & 33.00 & 33.55 & 34.11 & 34.67 & 35.24 & 35.82 \\
\hline
\end{tabular}




\begin{tabular}{|l|l|l|l|l|l|l|l|l|l|l|l|l|}
\hline $50-54$ & 31.97 & 32.47 & 32.98 & 33.50 & 34.02 & 34.55 & 28.67 & 29.20 & 29.73 & 30.27 & 30.82 & 31.38 \\
\hline $55-59$ & 27.56 & 28.05 & 28.54 & 29.04 & 29.55 & 30.06 & 24.52 & 25.02 & 25.52 & 26.04 & 26.56 & 27.09 \\
\hline $60-64$ & 23.33 & 23.80 & 24.27 & 24.75 & 25.24 & 25.74 & 20.62 & 21.09 & 21.57 & 22.06 & 22.55 & 23.06 \\
\hline $65-69$ & 19.32 & 19.76 & 20.21 & 20.67 & 21.13 & 21.61 & 16.99 & 17.43 & 17.87 & 18.33 & 18.80 & 19.28 \\
\hline $70-74$ & 15.65 & 16.06 & 16.47 & 16.90 & 17.33 & 17.78 & 13.73 & 14.14 & 14.55 & 14.98 & 15.42 & 15.87 \\
\hline $75-79$ & 12.34 & 12.71 & 13.08 & 13.47 & 13.87 & 14.28 & 10.84 & 11.21 & 11.59 & 11.97 & 12.38 & 12.79 \\
\hline $80+$ & 9.33 & 9.65 & 9.98 & 10.32 & 10.67 & 11.04 & 8.31 & 8.63 & 8.96 & 9.30 & 9.66 & 10.03 \\
\hline
\end{tabular}

Table 5.3: Forecasted values of life expectancy for 2020-2025 (I), 2025-2030 (II), 2030-2035 (III), $2035-$ 2040 (IV), 2040-2045 (V) and 2045-2050 (VI)

\begin{tabular}{|c|c|c|c|c|c|c|}
\hline \multirow{2}{*}{$\begin{array}{c}\text { Age } \\
\text { Group }\end{array}$} & \multicolumn{3}{|c|}{ Female } & \multicolumn{3}{|c|}{ Male } \\
\hline & I & III & VI & I & III & VI \\
\hline 0 & $77.33-78.79$ & $76.97-82.19$ & $75.09-88.05$ & $72.65-73.96$ & $72.83-77.40$ & $71.95-83.01$ \\
\hline $1-4$ & $77.67-78.91$ & $77.37-81.91$ & $75.82-87.36$ & $73.27-74.34$ & $73.41-77.29$ & $72.70-82.40$ \\
\hline $5-9$ & $74.29-75.41$ & $74.02-78.2$ & $72.66-83.47$ & $69.97-70.92$ & $70.09-73.63$ & $69.47-78.53$ \\
\hline $10-14$ & $69.47-70.57$ & $69.22-73.3$ & $67.90-78.52$ & $65.20-66.13$ & $65.32-68.77$ & $64.72-73.60$ \\
\hline $15-19$ & $64.58-65.66$ & $64.33-68.36$ & $63.03-73.55$ & $60.35-61.26$ & $60.47-63.86$ & $59.88-68.65$ \\
\hline $20-24$ & $59.72-60.78$ & $59.47-63.44$ & $58.21-68.58$ & $55.57-56.46$ & $55.69-59.00$ & $55.11-63.73$ \\
\hline $25-29$ & $54.90-55.93$ & $54.66-58.54$ & $53.44-63.63$ & $50.89-51.74$ & $51.00-54.21$ & $50.45-58.85$ \\
\hline $30-34$ & $50.11-51.11$ & $49.88-53.67$ & $48.69-58.70$ & $46.25-47.07$ & $46.36-49.47$ & $45.83-54.01$ \\
\hline $35-39$ & $45.35-46.33$ & $45.13-48.82$ & $43.99-53.78$ & $41.65-42.44$ & $41.75-44.76$ & $41.24-49.20$ \\
\hline $40-44$ & $40.66-41.60$ & $40.44-44.03$ & $39.34-48.91$ & $37.10-37.86$ & $37.20-40.11$ & $36.71-44.44$ \\
\hline $45-49$ & $36.04-36.95$ & $35.83-39.32$ & $34.77-44.10$ & $32.64-33.37$ & $32.74-35.53$ & $32.27-39.75$ \\
\hline $50-54$ & $31.53-32.41$ & $31.33-34.71$ & $30.30-39.40$ & $28.33-29.02$ & $28.42-31.09$ & $27.98-35.18$ \\
\hline $55-59$ & $27.14-27.99$ & $26.94-30.21$ & $25.96-34.79$ & $24.19-24.85$ & $24.28-26.82$ & $23.86-30.77$ \\
\hline $60-64$ & $22.93-23.74$ & $22.74-25.89$ & $21.81-30.33$ & $20.31-20.93$ & $20.39-22.80$ & $20.00-26.59$ \\
\hline $65-69$ & $18.94-19.71$ & $18.77-21.75$ & $17.89-26.02$ & $16.7-17.28$ & $16.78-19.04$ & $16.41-22.64$ \\
\hline $70-74$ & $15.30-16.01$ & $15.13-17.91$ & $14.33-21.98$ & $13.47-14.00$ & $13.54-15.64$ & $13.20-19.05$ \\
\hline $75-79$ & $12.02-12.66$ & $11.88-14.40$ & $11.16-18.20$ & $10.61-11.09$ & $10.67-12.58$ & $10.36-15.75$ \\
\hline $80+$ & $9.06-9.61$ & $8.94-11.14$ & $8.33-14.62$ & $8.11-8.52$ & $8.16-9.84$ & $7.90-12.73$ \\
\hline
\end{tabular}

Table 5.4: Confidence Intervals for life expectancy for 2020-2025 (I), 2030-2035 (III), and 2045-2050 (VI) 


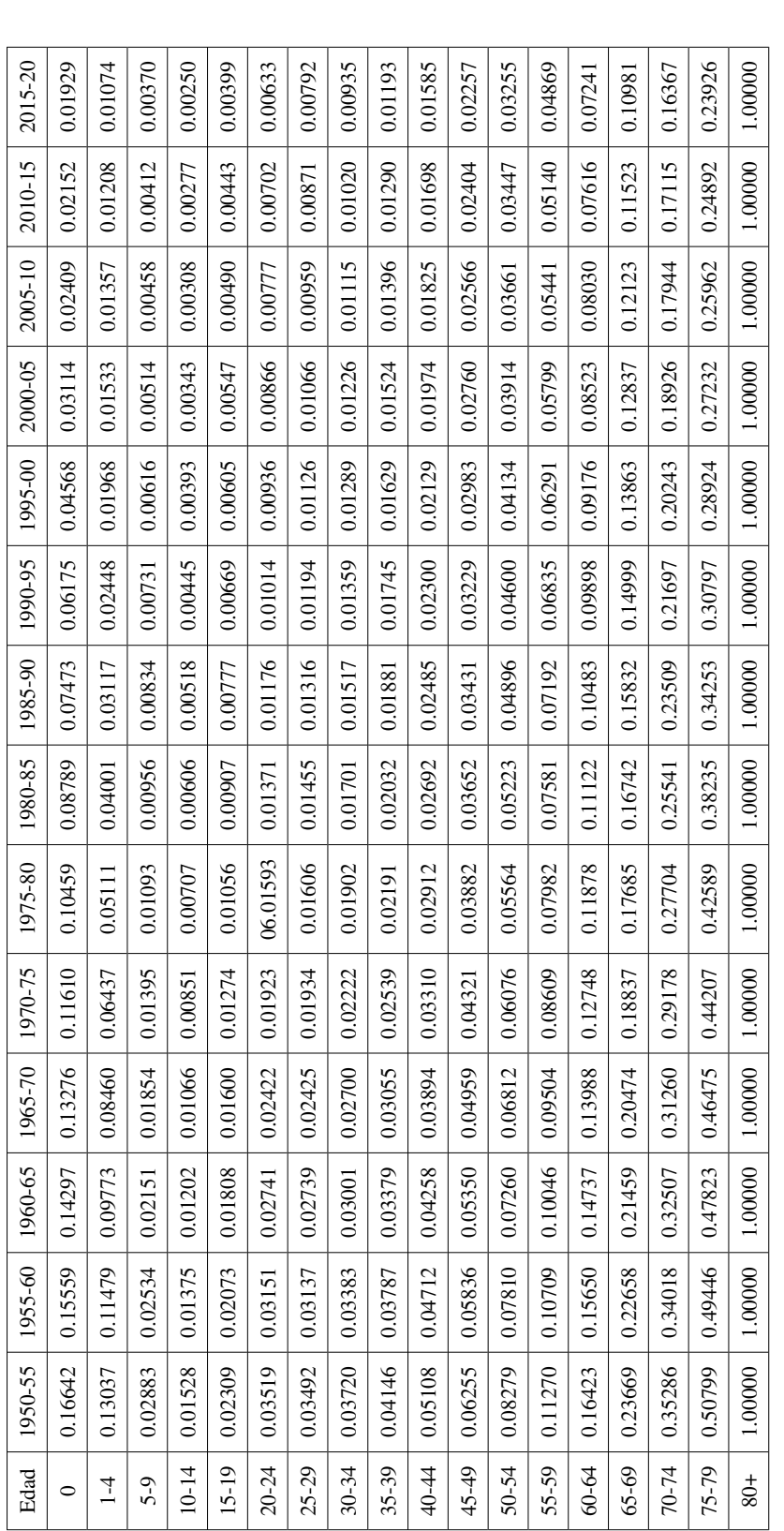

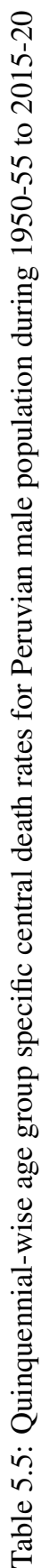




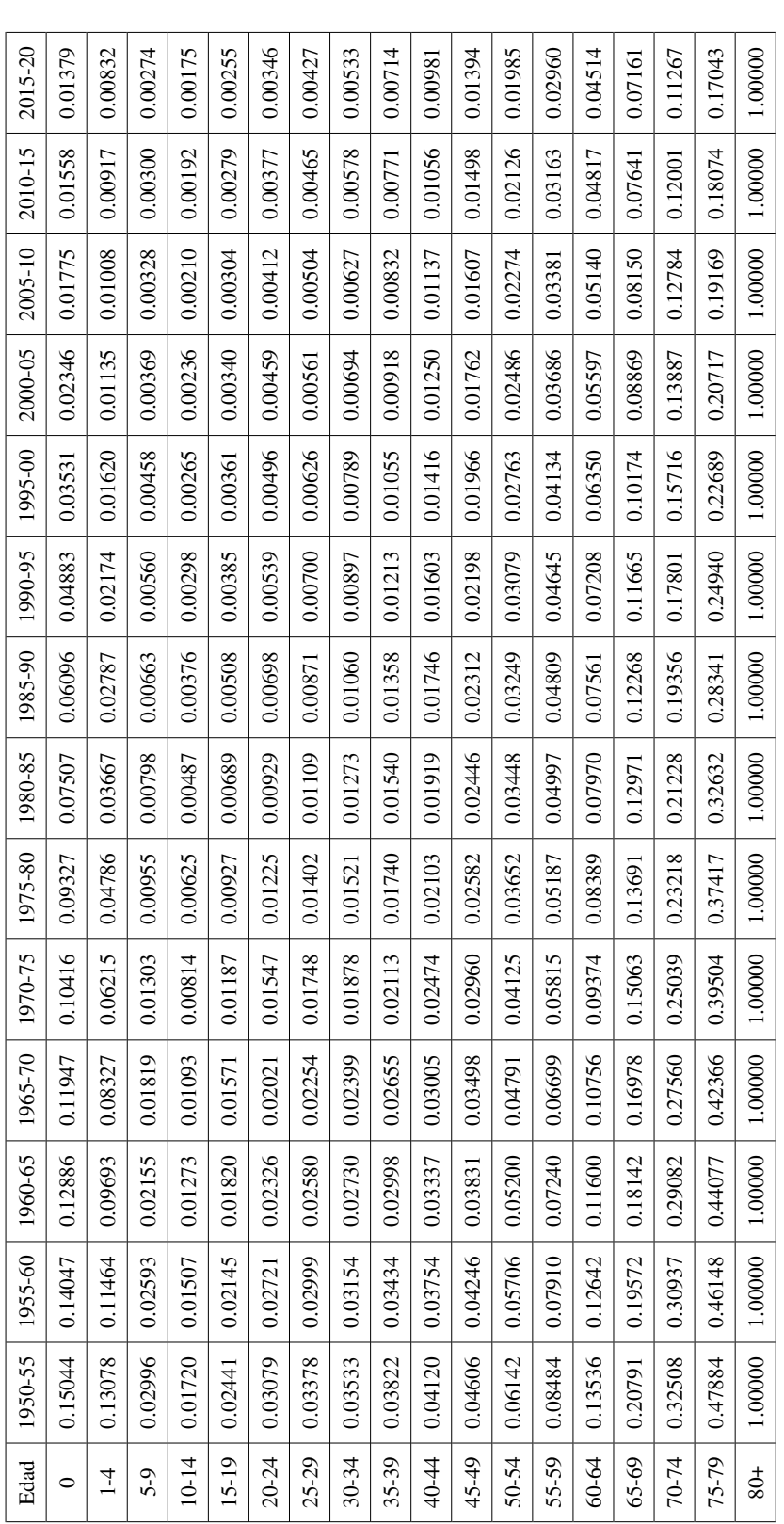

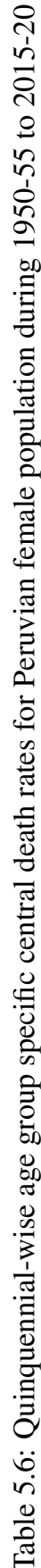


6. Conclusions. In this paper we illustrate the performance of the LC approach to modeling the central mortality rates of Peruvian population. The principal objective of this study is to estimate the model parameters in order to predict future values of central mortality rates as well as future life expectancy. The data for central mortality rates is available only for 14 five-years periods (census data), from 1950 to 2017. As mentioned above, these predictions are utilized by life insurance companies and annuity providers for their pricing calculations. The results, presented in this article demonstrate a very good fit of the model to the data. On the other hand, the confidence intervals for life expectancy, presented in Table 5.4 are somewhat wide for more distant periods, especially for the male population. This can probably be explained by a large variability of the mortality index predictions due to a shortness of the series of the mortality index (recall that in our case it is only 14). Since the insurance companies are interested in long term predictions, controlling the width of confidence intervals is generally of great importance to them. In order to solve the problem of large confidence intervals, one can "borrow" information from the males mortality tables when estimating the model parameters in the female population, and vice versa. Another interesting extension of the research is studying the performance of the LC model in terms of its predictive characteristics by employing the bootstrap methods. The authors are working in these directions.

7. Acknowledgements. The views presented in this work are those of the authors and do not represent the official position of the institutions that the authors are or were affiliated with. JCH was partially supported by CONCYTEC- FONDECYT (contract 232-2019 and 427-2019).

\section{ORCID and License}

J. Cerda-Hernández https: / / orcid.org/0000-0002-9297-5694

A. Sikov https://orcid.org/0000-0001-9869-5952

This work is licensed under the Creative Commons - Attribution 4.0 International (CC BY 4.0)

\section{References}

[1] Baran S, Gall J, Ispany M and Pap M. Forecasting Hungarian Mortality Rates Using The Lee-Carter Method. Acta Economica; (2007). 57, 21-34.

[2] Booth H, Maindonald J and Smith L. Applying Lee-Carter under conditions of variable mortality decline. Population studies. 2002; 56(3): 325-336.

[3] Brockwell PJ and Davis RA. Time Series: Theory and Methods. New York: Springer; 2013.

[4] Cairns AJ, Blake D and Dowd K. A Two Factor Model for Stochastic Mortality with Parameter Uncertainty: Theory and Calibration. Journal of Risk and Insurance. 2006; 73(4):687-718.

[5] Hamilton JD. Time Series Analysis. Princeton: Princeton Univ. Press; 1994.

[6] Horiuchi S and Wilmoth JR. Deceleration in the age pattern of mortality at older ages. Demography. 1998; 35(4):391-412.

[7] Khamladze EV. Statistical Methods With Application to Demography and Life Insurance. New York: Taylor and Franciss Group; 2013.

[8] Koissi MC and Shapiro A. The Lee-Carter model under the condition of variables age-specific parameters. 43rd Actuarial Research Conference, Canada, Regina, 2008.

[9] Lawson C and Hanson R. (1974). Solving Least Squares Problems. Elglewood Cliffs, N.J. Prentice-Hall; 1974.

[10] Lee RD and Carter LR. (1992) Modeling and forecasting US mortality. Journal of the American statistical association. 1992; 87(419):659-671.

[11] Lee RD and Rofman R. (1994). Modeling and forecasting mortality in Chile. Notas. 1994; 22(59):182-213.

[12] Lin J. Changing Kinship Structure and its Implications for Old-Age Support in Urban and Rural China. Population Studies. 1995; 49(1): 127- 145 .

[13] Li SH and Chan WS. (2004). Estimation of Complete period life tables for singaporeans. Journal of Actuarial Practice. 2004; 11:129-146.

[14] Ngataman N, Ibrahim RI and Yusuf MM. Forecasting the Mortlaity Rates of Malaysian Population Using Lee-Carter Method. American Institue of Physics; 2016.

[15] Rajendra NC and Ramkrishna LS. Modeling and Forecasting Mortality Using the Lee-Carter Model for Indian Population Based on Decade-wise Data Sri Lankan Journal of Applied Statistics. 2016; 17-1.

[16] Renshaw AE and Haberman S. (2006) A cohort-based extension to the LeeCarter model for mortality reduction factors. Insurance: Mathematics and Economics. 2006; 38(3):556-570.

[17] Shores T. Applied Linear Algebra and Matrix Analysis. Heildelberg: Springer Verlag. 2004.

[18] Thatcher AR. The long-term pattern of adult mortality and the highest attained age. J R Stat Soc Ser A Stat Soc. 1999; 162(1):543.

[19] Tuljapurkar S, Nan L and Boe C. (2000). A universal pattern of mortality decline in the G7 countries. Nature. 2000; 405:789-792.

[20] Wilmoth JR. (1993) Computational Methods for Fitting and Extrapolating the Lee-Carter Model of Mortality Change. University of California, Berkeley. USA. Technical Report; 1993.

[21] Wilmoth JR. (1996). Mortality Projections for Japan: A Comparison of Four Methods. Oxford University Press, New York. Health and Mortality among Elderly Population. In: Caselli, G., Lopez, A. (Eds.); 1996.

[22] Yadav A, Yadav S and Kesarwani R. (2012). Decelerating Mortality Rates in Older Ages and its Prospects through Lee-Carter Approach. PLoS ONE. 2012; 7(12). 